\section{(2) OPEN ACCESS}

- Additional material is published online only. To view please visit the journal online (http://dx.doi.org/10.1136/ heartjnl-2019-316219).

${ }^{1}$ Division of Cardiovascular Diseases, Korea National Institute of Health, Cheongju, Chungcheongbuk-do, The Republic of Korea IInternal Medicine, Seoul National University Bundang Hospital, Seongnam, Gyeonggi, The Republic of Korea

\section{Correspondence to}

Dr Won-Ho Kim, Division of Cardiovascular Diseases, Korea National Institute of Health, Cheongju 28159, Korea (the Republic of); jhkwh@nih.go.kr and Dr Dong-Ju Choi, Internal Medicine, Seoul National University Bundang Hospital, Seongnam, Gyeonggi, The

Republic of Korea; djchoi@snubh.org

MKS and JJP contributed equally.

Received 10 November 2019 Revised 28 February 2020 Accepted 8 March 2020 Published Online First 27 April 2020

\title{
Impact of atrial fibrillation in patients with heart failure and reduced, mid-range or preserved ejection fraction
}

\author{
Mi Kyoung Son, ${ }^{1}$ Jin Joo Park, ${ }^{2}$ Nam-Kyoo Lim, ${ }^{1}$ Won-Ho Kim (ㄷ, , Dong-Ju Choi (i) ${ }^{2}$
}

\begin{abstract}
Objective To determine the prognostic value of atrial fibrillation (AF) in patients with heart failure (HF) and preserved, mid-range or reduced ejection fraction (EF). Methods Patients hospitalised for acute HF were enrolled in the Korean Acute Heart Failure registry, a prospective, observational, multicentre cohort study, between March 2011 and February 2014. HF types were defined as reduced $\mathrm{EF}$ (HFrEF, LVEF $<40 \%$ ), mid-range EF (HFmrEF, LVEF $40 \%-49 \%$ ) or preserved EF (HFpEF, LVEF $\geq 50 \%$ ).
\end{abstract}

Results Of 5414 patients enrolled, HFrEF, HFmrEF and HFpEF were seen in 3182 (58.8\%), 875 (16.2\%) and 1357 (25.1\%) patients, respectively. The prevalence of AF significantly increased with increasing EF (HFrEF 28.9\%, HFmrEF 39.8\%, HFpEF 45.2\%; p for trend <0.001). During follow-up (median, 4.03 years; IQR, 1.39-5.58 years), 2806 (51.8\%) patients died. The adjusted HR of AF for all-cause death was $1.06(0.93-1.21)$ in the HFrEF, $1.10(0.87-1.39)$ in the HFmrEF and 1.22 (1.021.46) in the HFpEF groups. The HR for the composite of all-cause death or readmission was 0.97 (0.87-1.07), $1.14(0.93-1.38)$ and $1.03(0.88-1.19)$ in the HFrEF, HFmrEF and HFpEF groups, respectively, and the HR for stroke was $1.53(1.03-2.29), 1.04(0.57-1.91)$ and 1.90 $(1.13-3.20)$, respectively. Similar results were observed after propensity score matching analysis.

Conclusions AF was more common with increasing EF. AF was seen to be associated with increased mortality only in patients with $\mathrm{HFpEF}$ and was associated with an increased risk of stroke in patients with HFrEF or HFpEF. Trial registration number NCT01389843

\section{INTRODUCTION}

Heart failure (HF) and atrial fibrillation (AF) are leading causes of mortality and ischaemic stroke. ${ }^{1-5}$ The prevalence of AF in patients with $\mathrm{HF}$ and the mortality rate in these patients vary according to ethnicity. ${ }^{6}$ Their prevalence is also expected to increase due to a growing burden of risk factors, such as an ageing population, hypertension, obesity, diabetes mellitus and ischaemic heart disease. ${ }^{6}$

Because AF and HF share common risk factors, they frequently coexist, and patients with both AF and HF have a worse prognosis than those with either of these conditions alone. ${ }^{7-9}$ However, the prognostic implications of $\mathrm{AF}$ in patients with HF remain controversial. The majority of current data suggest that $\mathrm{AF}$ is associated with increased mortality in patients with HF and preserved ejection fraction and in those with reduced ejection fraction. ${ }^{10-17}$ By contrast, the HF long-term registry of the European Society of Cardiology showed that $\mathrm{AF}$ was not associated with poor outcomes in patients with HFrEF. ${ }^{18}$

There are similarities and differences in HF across different ethnicities. ${ }^{19}$ The Korean Acute Heart Failure (KorAHF) registry is the largest prospective registry in Korea and includes all patients hospitalised for HF. Here, this database has been used to investigate the clinical characteristics and prognostic impact of AF in Korean patients according to the HF subtypes defined by ejection fraction (EF).

\section{METHODS}

\section{Data source}

The KorAHF registry, supported by the Korea National Institute of Health, is a prospective, observational, multicentre cohort registry that enrolled 5625 patients hospitalised with acute $\mathrm{HF}$ at 10 tertiary university hospitals across the country from March 2011 to February 2014. ${ }^{19} 20$ Patients with signs or symptoms of HF and either lung congestion, objective findings of left ventricular systolic dysfunction or structural heart disease were eligible for inclusion in the study and were scheduled for follow-up until the end of 2018. The study design, data validation and an interim analysis were reported previously. ${ }^{19}{ }^{20}$ All patients were followed up for at least 3 years, and data, including cause of death or readmission and various clinical measurements, were collected until December 2018. Mortality data for patients lost to follow-up were obtained from national death records.

\section{Study variables}

Left ventricular EF (LVEF) was measured using the Simpson's biplane method at the acute HF hospitalisation. If the Simpson's method was not possible, then LVEF was assessed using M-mode or visual estimation. Patients with HF were categorised as reduced EF (HFrEF) (EF < 40\%), midrange $\mathrm{EF}$ (HFmrEF) (EF 40\%-49\%) or preserved $\mathrm{EF}$ (HFpEF) (EF $\geq 50 \%)$. The presence of $\mathrm{AF}$ was confirmed by ECG during the index admission. The primary outcomes were all-cause mortality, the composite of all-cause mortality and readmission for HF, cardiovascular (CV) mortality, and stroke during the follow-up period.

Most hospitals participating in this registry routinely collected data on either brain natriuretic
To cite: Son MK, Park JJ, Lim N-K, et al. Heart 2020;106:1160-1168 
peptide (BNP) or N-terminal prohormone of brain natriuretic peptide (NT-proBNP) levels. A composite variable was created by combining the two individual variables as follows: (1) BNP $>900$ or NT-proBNP $>5000 \mathrm{pg} / \mathrm{mL}$ and (2) other.

\section{Statistical analysis}

Baseline characteristics according to AF and HF type at the time of index admission were captured. Continuous variables were expressed as mean $\pm \mathrm{SD}$ and compared using t-tests; categorical variables were expressed as frequency (percentage) and compared using $\chi^{2}$ tests. The prevalence of AF in patients with HFrEF, HFmrEF and HFpEF, according to age and sex, was calculated, and $\mathrm{p}$ for trend was obtained using the CochranArmitage method.

The event rates of outcomes (all-cause mortality, the composite of all-cause mortality or readmission, CV mortality, and stroke) were reported as the number of patients per 1000 person-years. Kaplan-Meier curves were created for time to outcomes according to the presence of $\mathrm{AF}$ in the $\mathrm{EF}$ group; survival distributions were compared using the log-rank test when proportional assumption was met, otherwise using the Breslow's (the generalised Wilcoxon) test. ${ }^{21}$ The proportional hazards assumption was tested using the scaled Schoenfeld residuals. $^{22}$

The association between AF and every outcome in each EF group was determined by the Cox proportional hazard regression model with adjustment for potential confounders, including sex, age, smoking status, alcohol consumption, medical history (New York Heart Association functional class, hypertension, diabetes mellitus, ischaemic heart disease, renal failure, cancer, prior stroke), heart rate, systolic blood pressure, diastolic blood pressure, body mass index, laboratory examination (white cell count, glycated haemoglobin, potassium, sodium, haemoglobin, blood urea nitrogen, creatinine, BNP or NT-proBNP), and medications before or at admission or at discharge (use of ACE inhibitor or angiotensin receptor blockers, beta-blockers, aldosterone antagonist, nitrate, diuretic, anticoagulant, antiplatelet and statin). Considering the high mortality rate of patients with acute HF, we also performed the competing risk regression analysis using the Fine and Gray model. ${ }^{23}$

To reduce the possibility of biased effect estimates in this study, propensity score matching analyses were performed. A propensity score according to AF status was estimated using variables known to be related to both the group assignments and the outcome variables: demographics, medical history, examinations, laboratory examination and medications.

All statistical tests were two-tailed and $\mathrm{p}$ values $<0.05$ were considered statistically significant. All statistical analyses were performed using SAS V.9.4 software and R V.3.5.3 (R Foundation, Vienna, Austria). R packages (http://cran. r-project.org) of 'survival', 'MatchIt' and 'cmprsk' were used to conduct the survival analysis, construct the matched cohort and conduct competing risk regression analysis, respectively.

\section{Patient and public involvement}

This research was done without patient involvement. Patients were not invited to comment on the study design and were not consulted to develop patient relevant outcomes or interpret the results. Patients were not invited to contribute to the writing or editing of this document for readability or accuracy.

\section{RESULTS}

\section{Baseline characteristics}

Of 5625 patients enrolled in the KorAHF registry, 211 patients with missing EF information were excluded. Of the remaining 5414 patients, 1883 (34.8\%) exhibited AF, as confirmed by ECG at admission. According to the EF, 3182 (58.8\%), 875 (16.2\%) and $1357(25.1 \%)$ patients were classified as having HFrEF, HFmrEF and HFpEF, respectively (figure 1).

The baseline characteristics of patients with and without AF differed according to the HF type. In general, patients with AF were older and less likely to be diabetic or current smokers than those without AF. Regarding HF type, the age and proportion of the female patients increased with EF (table 1).

The prevalence of AF in the HFrEF, HFmrEF and HFpEF groups was $28.9 \%, 39.8 \%$ and $45.2 \%$, respectively. The prevalence of $\mathrm{AF}$ according to age and sex significantly increased with increasing EF (figure 2).

\section{Clinical outcomes}

During the follow-up period (median, 4.03 years; IQR, 1.395.58 years), 2806 (51.8\%) patients died; the all-cause mortality did not differ between patients with AF (969 patients, 51.5\%) and those without AF (1837 patients, 52.0\%; $\mathrm{p}=0.692$ ).

When stratifying the patients according to HF type, patients with AF had lower all-cause mortality in the HFrEF group (129.46 vs 145.03 events/1000 patient-years in patients with $\mathrm{AF})$, but all-cause mortality was higher among patients with AF in the HFpEF group (150.42 vs 139.68 events/1000 patientyears; table 2). Similar findings were observed for CV mortality and the composite of all-cause mortality and readmission. The event rate for stroke was higher in patients with $\mathrm{AF}$ than in those without AF across all HF types (HFrEF: 16.60 vs 11.63 events/1000 patient-years, respectively; HFmrEF: 28.06 vs 22.00 events/1000 patient-years, respectively; HFpEF: 25.57 vs 12.89 events/1000 patient-years, respectively).

In the Kaplan-Meier survival analysis, all-cause mortality and the composite of all-cause mortality or readmission did not differ between the groups. By contrast, there was a significant difference for CV mortality and stroke events. Patients with HFrEF without $\mathrm{AF}$ had the highest $\mathrm{CV}$ mortality risk, while patients with HFpEF without AF had the lowest. Regarding stroke, patients with $\mathrm{HFrEF}$ without AF had the lowest stroke event risk and those with HFmrEF with AF had the highest (figure 3).

After multivariable adjustment for covariates, AF was significantly associated with increased all-cause mortality (HR, 1.11; $95 \%$ CI 1.01 to 1.22 ), while the statistical significance

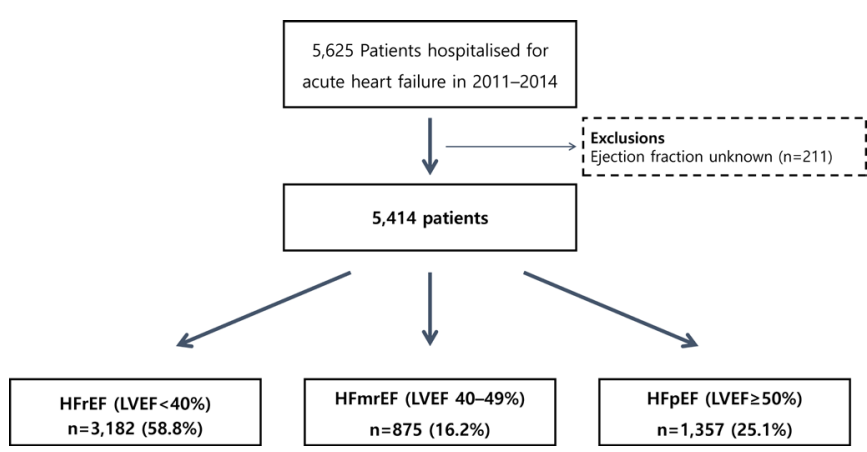

Figure 1 Study population flow chart. HFmrEF, heart failure with mid-range ejection fraction; $\mathrm{HFpEF}$, heart failure with preserved ejection fraction; HFrEF, heart failure with reduced ejection fraction; LVEF, left ventricular ejection fraction. 


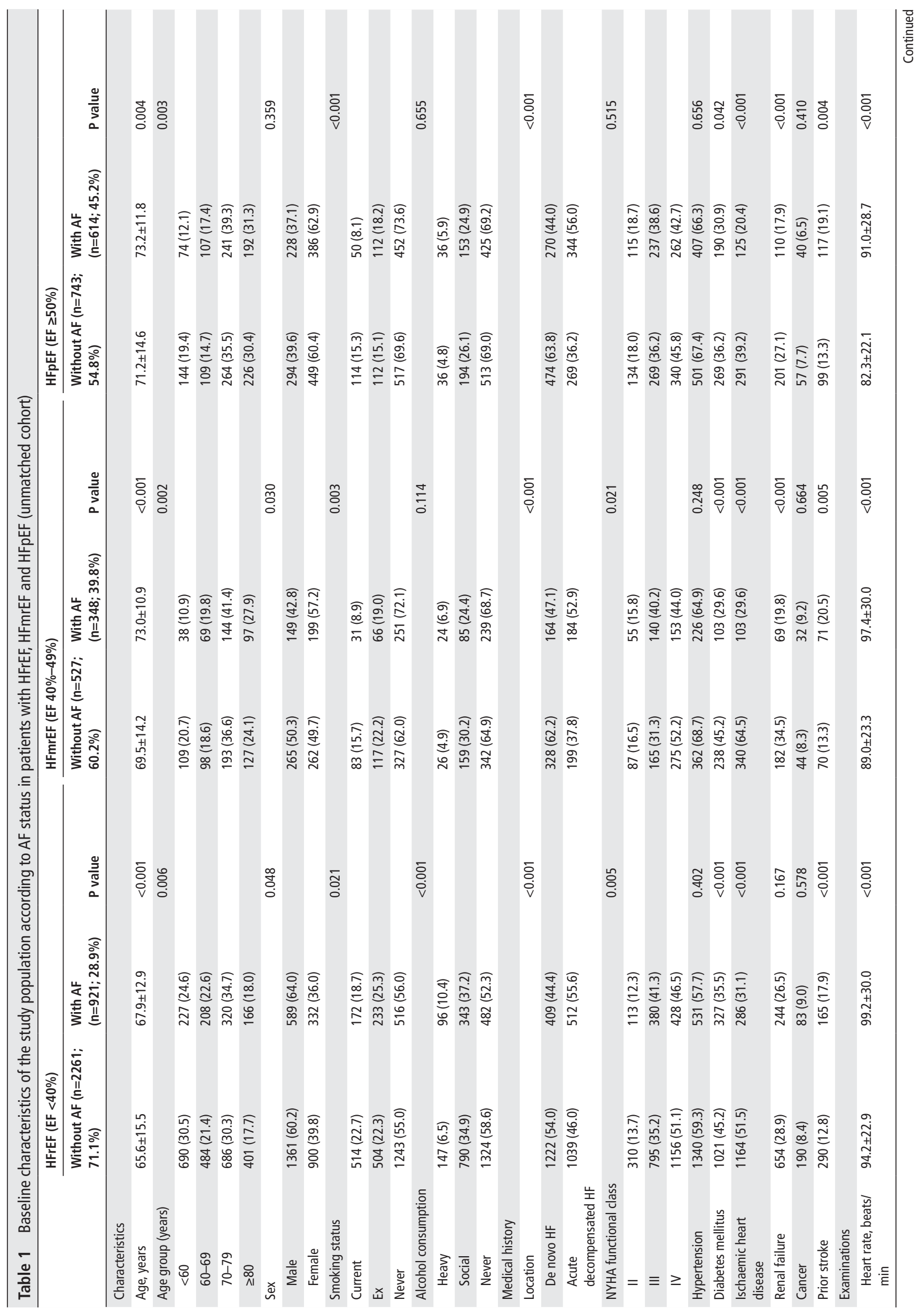




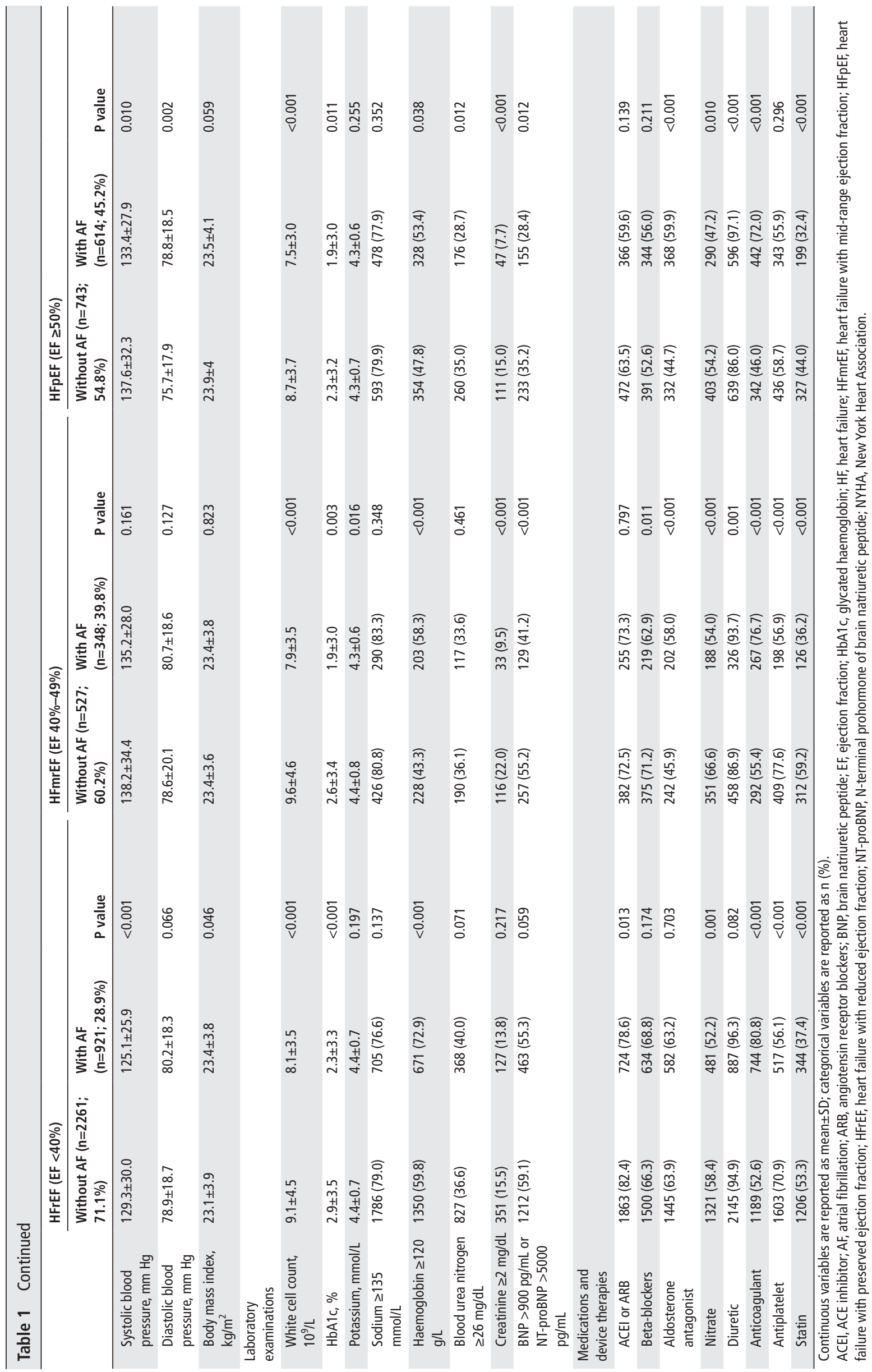




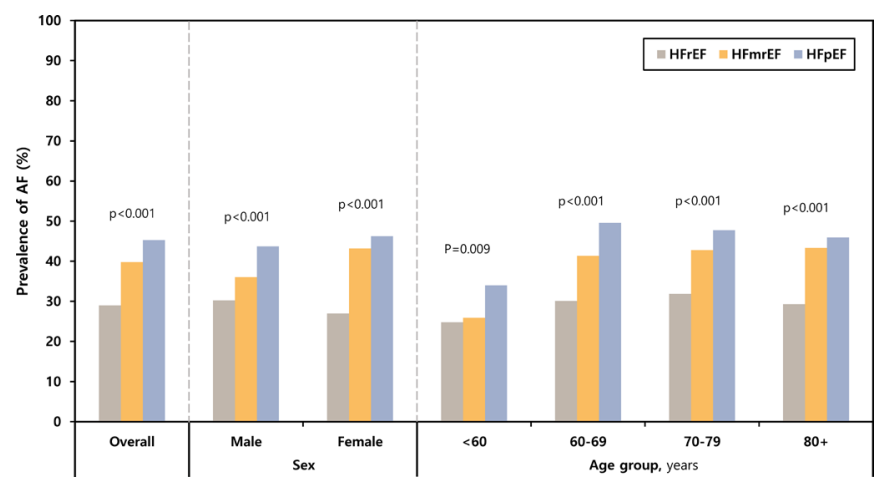

Figure 2 Prevalence of AF in patients with HFrEF, HFmrEF and HFpEF according to age and sex; $p$ value for trend. $A F$, atrial fibrillation; $H F m r E F$, heart failure with mid-range ejection fraction; HFpEF, heart failure with preserved ejection fraction; HFrEF, heart failure with reduced ejection fraction.

disappeared with propensity score matching (HR, 1.05; 95\% CI 0.94 to 1.16; figure 4). By contrast, AF was associated with a 1.6-fold increased risk for stroke in all patients. Analysis of the data according to HF type showed that AF was associated with increased all-cause and CV mortality in patients with HFpEF (HR, 1.22, 95\% CI 1.02 to 1.46 for all-cause mortality; HR, 1.55 , 95\% CI 1.06 to 2.27 for CV mortality) but not in those with HFrEF and HFmrEF. Regarding stroke, AF was associated with an increased risk for stroke in patients with HFrEF (HR, 1.53; 95\% CI 1.03 to 2.29) and HFpEF (HR, $1.90 ; 95 \%$ CI 1.13 to 3.20 ; online supplementary files 1-1 to 1-4). Regarding stroke and CV mortality, these are similar to the results obtained from the competing risk analysis after considering all-cause death from causes other than outcomes of interest as a competing risk (table 3 ).

Under stratification by rhythm, in patients without AF, there was no significant difference in all-cause mortality, the composite of all-cause mortality and hospitalisation for $\mathrm{HF}$, and stroke between the HF subtypes. Similar findings were observed for patients with AF (online supplementary file 2). The similar clinical outcomes of the three HF phenotypes imply that the differential effect of AF according to HF phenotypes was mainly driven by AF per se, not by HF phenotypes ( $p$ for interaction 0.174 for all-cause mortality, 0.136 for the composite of mortality or readmission for HF, 0.220 for CV mortality, and 0.382 for stroke, respectively).

\section{Subgroup analysis}

The event rates for mortality, the composite of mortality or readmission, and $\mathrm{CV}$ mortality were higher in patients with renal failure with and without AF in each of the EF groups (online supplementary files 3-1 to 3-3). The event rate for stroke was higher in patients with prior stroke in each of the EF groups (online supplementary file 3-4). In the subgroup analysis of CV mortality, there was a significant interaction with diabetes in the HFpEF group, and patients without diabetes appeared to be more affected by AF ( $p$ for interaction=0.033). Regarding stroke, the interaction was significant with renal failure in patients with HFpEF ( $p$ for interaction=0.019); the effect of $\mathrm{AF}$ on stroke was higher in patients without renal failure. By contrast, regarding mortality and stroke, there was no significant interaction of drug therapy with AF.

\section{Propensity score matching}

After matching, the baseline characteristics were well balanced between patients with and without $\mathrm{AF}$ in each of the three EF groups (standardised mean difference $<0.25$; online supplementary file 4). As seen in the unmatched cohort, there was a significant difference in stroke event and CV mortality rates between patients with and without AF across the HF types, but all-cause mortality and the composite of all-cause mortality or readmission did not differ between the groups (online supplementary file 5). AF was also significantly associated with an increased risk for all-cause and CV mortality in patients with HFpEF, and stroke in patients with HFpEF and HFrEF (figure 4). Regarding the AF types, the all-cause mortality and stroke did not differ between AF types (paroxysmal AF vs permanent or persistent $\mathrm{AF}$ ) in the propensity score matched cohort (online supplementary file 6).

Table 2 Event rates per 1000 person-years according to AF status and ejection fraction group

\begin{tabular}{|c|c|c|c|c|}
\hline \multirow[b]{2}{*}{ Outcomes } & \multicolumn{2}{|l|}{ Without AF } & \multicolumn{2}{|l|}{ With AF } \\
\hline & Cases/person-years & IR $(95 \% \mathrm{Cl})$ & Cases/person-years & IR $(95 \% \mathrm{Cl})$ \\
\hline \multicolumn{5}{|c|}{ All-cause mortality } \\
\hline HFrEF & $1167 / 8046.49$ & 145.03 (136.83 to 153.47$)$ & $442 / 3414.18$ & $129.46(117.67$ to 141.80$)$ \\
\hline HFmrEF & $282 / 1908.76$ & 147.74 (131.00 to 165.47$)$ & $189 / 1279.76$ & 147.68 (127.38 to 169.47$)$ \\
\hline HFpEF & $388 / 2777.88$ & 139.68 (126.12 to 153.91$)$ & $338 / 2247.09$ & 150.42 (134.81 to 166.87$)$ \\
\hline \multicolumn{5}{|c|}{ Composite of all-cause mortality and readmission } \\
\hline HFrEF & $1819 / 3705.35$ & 490.91 (468.61 to 513.73 ) & $713 / 1676.43$ & 425.31 (394.66 to 457.09$)$ \\
\hline HFmrEF & $414 / 935.75$ & 442.43 (400.83 to 486.05$)$ & $289 / 585.46$ & 493.63 (438.35 to 552.14$)$ \\
\hline HFpEF & $596 / 1237.30$ & 481.69 (443.79 to 521.12$)$ & $492 / 1121.42$ & 438.73 (400.81 to 478.33 ) \\
\hline \multicolumn{5}{|c|}{ CVD mortality } \\
\hline HFrEF & $389 / 8046.49$ & 48.34 (43.66 to 53.26$)$ & $141 / 3414.18$ & $41.30(34.76$ to 48.39$)$ \\
\hline HFmrEF & 70/1908.76 & $36.67(28.59$ to 45.75$)$ & $45 / 1279.76$ & $35.16(25.65$ to 46.16$)$ \\
\hline HFpEF & $81 / 2777.88$ & 29.16 (23.16 to 35.84$)$ & $80 / 2247.09$ & 35.60 (28.23 to 43.82$)$ \\
\hline \multicolumn{5}{|l|}{ Stroke } \\
\hline HFrEF & $91 / 7823.36$ & 11.63 (9.37 to 14.14$)$ & $54 / 3253.85$ & 16.60 (12.47 to 21.31$)$ \\
\hline HFmrEF & $40 / 1818.28$ & 22.00 (15.72 to 29.32$)$ & $34 / 1211.74$ & 28.06 (19.43 to 38.25$)$ \\
\hline HFpEF & $35 / 2715.16$ & 12.89 (8.98 to 17.50$)$ & $54 / 2111.44$ & 25.57 (19.21 to 32.83$)$ \\
\hline
\end{tabular}

$\mathrm{AF}$, atrial fibrillation; CVD, cardiovascular disease; HFmrEF, heart failure with mid-range ejection fraction; HFpEF, heart failure with preserved ejection fraction; HFrEF, heart failure with reduced ejection fraction; $I R$, incidence rate. 


\section{(A) All-cause mortality}

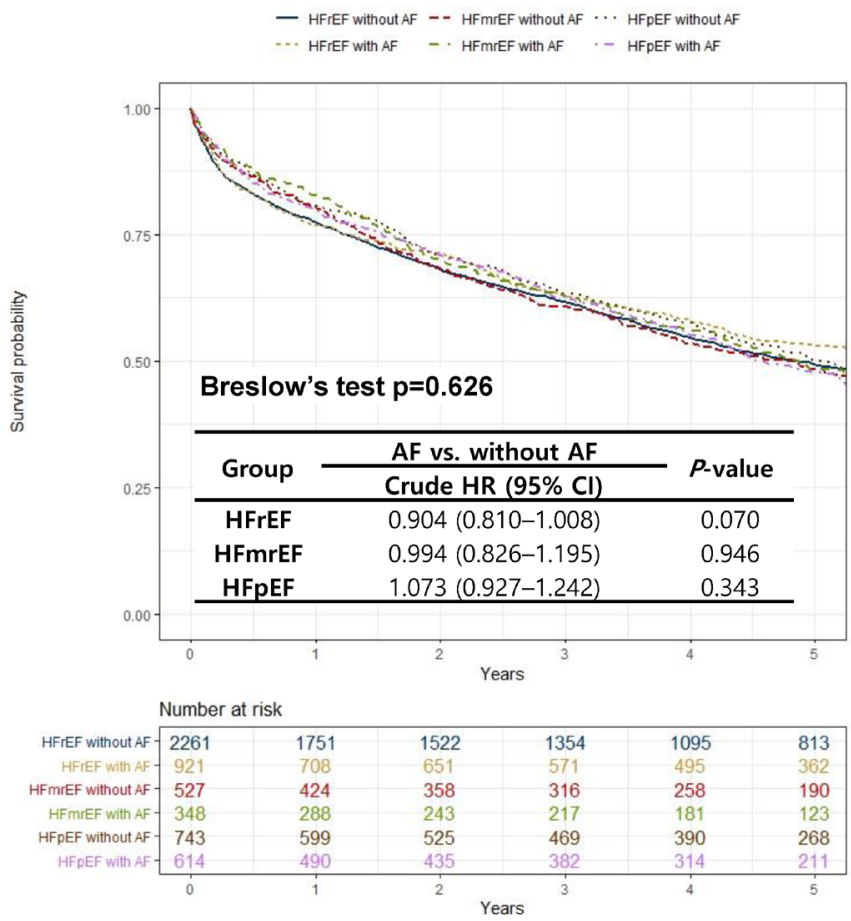

\section{(C) CVD mortality}

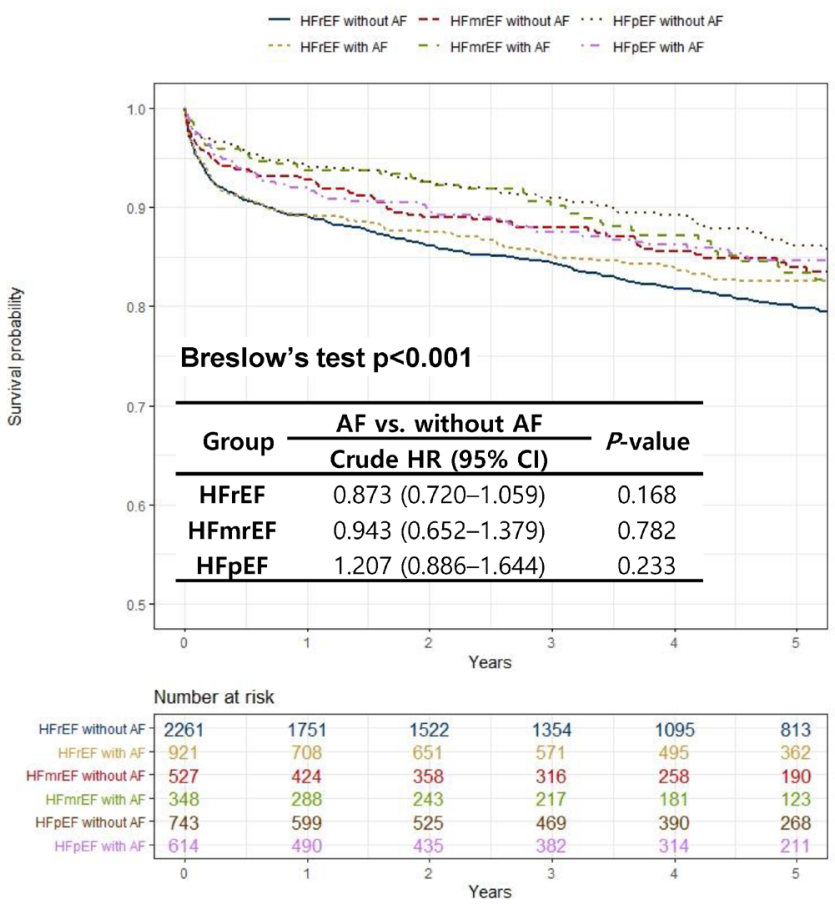

(B) All-cause mortality or rehospitalisation

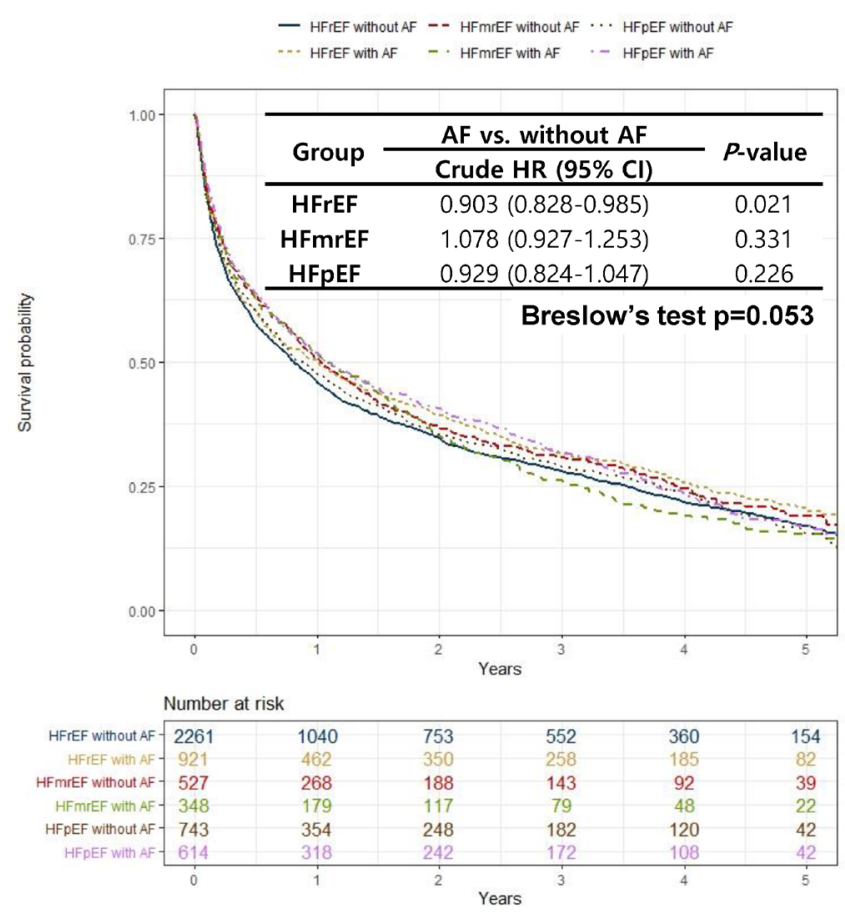

(D) Stroke

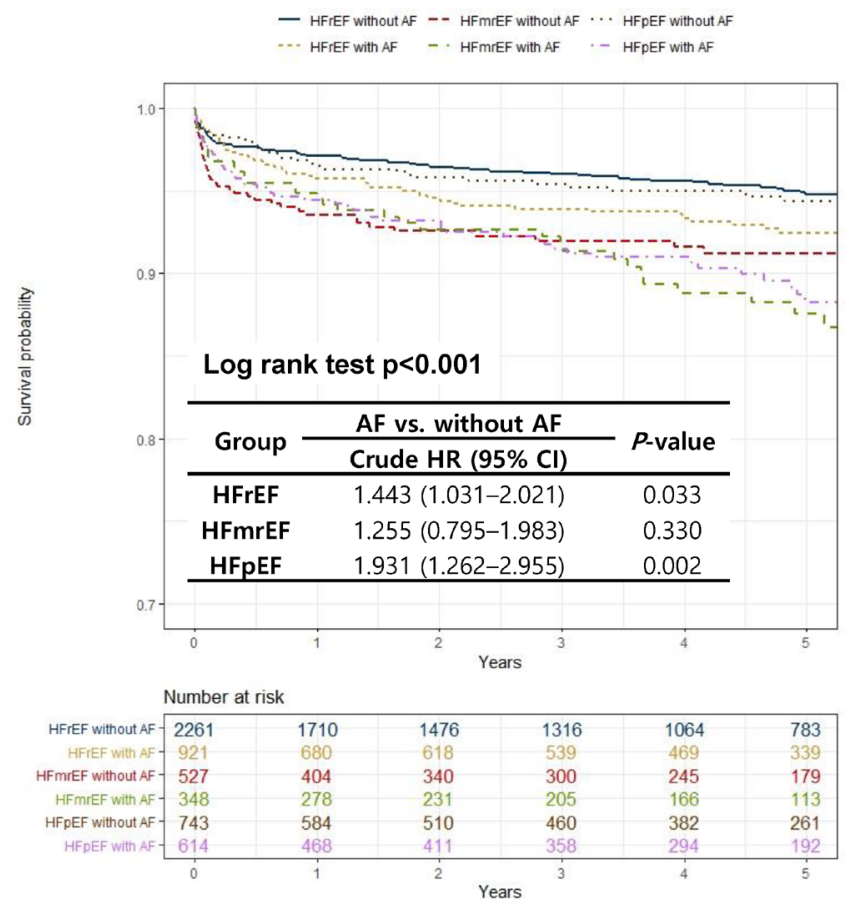

Figure 3 Kaplan-Meier curves for unmatched cohort. Data are stratified by the three ejection fraction groups and the presence or absence of AF for (A) all-cause mortality, (B) all-cause mortality or rehospitalisation, (C) CVD mortality and (D) stroke. AF, atrial fibrillation; CVD, cardiovascular disease; $\mathrm{HFmrEF}$, heart failure with mid-range ejection fraction; $\mathrm{HFpEF}$, heart failure with preserved ejection fraction; $\mathrm{HFrEF}$, heart failure with reduced ejection fraction.

\section{DISCUSSION}

In this study of patients with acute HF, the prevalence of $\mathrm{AF}$ increased with increasing EF. Surprisingly, no differences in mortality were seen between patients with and without AF.
However, when patients were stratified according to HF type, AF was associated with increased mortality in patients with HFpEF but not in those with HFrEF and HFmrEF. By contrast, $\mathrm{AF}$ was associated with an increased risk for stroke in all HF 
A. Unmatched Cohort

\begin{tabular}{|c|c|c|c|c|}
\hline EF group & Outcomes & with AF vs without AF & HR $(95 \% \mathrm{CI})$ & $P$-value \\
\hline \multirow[t]{4}{*}{ Overall } & All-cause mortality & 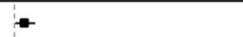 & $1.108(1.010-1.217)$ & 0.031 \\
\hline & All-cause mortality or rehospitalisation & & $1.008(0.935-1.088)$ & 0.831 \\
\hline & CVD mortality & & $1.025(0.859-1.223)$ & 0.783 \\
\hline & Stroke & $\longrightarrow$ & $1.600(1.222-2.094)$ & 0.001 \\
\hline \multirow[t]{4}{*}{ HFrEF } & All-cause mortality & - & $1.058(0.926-1.207)$ & 0.408 \\
\hline & All-cause mortality or rehospitalisation & 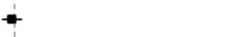 & $0.967(0.871-1.074)$ & 0.535 \\
\hline & CVD mortality & - & $0.908(0.716-1.150)$ & 0.423 \\
\hline & Stroke & $\longrightarrow$ & $1.534(1.028-2.290)$ & 0.036 \\
\hline \multirow[t]{4}{*}{ HFmrEF } & All-cause mortality & - & $1.098(0.866-1.392)$ & 0.441 \\
\hline & All-cause mortality or rehospitalisation & $\rightarrow-$ & $1.135(0.931-1.382)$ & 0.210 \\
\hline & CVD mortality & & $1.060(0.660-1.702)$ & 0.808 \\
\hline & Stroke & 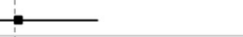 & $1.039(0.567-1.906)$ & 0.901 \\
\hline \multirow[t]{4}{*}{ HFpEF } & All-cause mortality & $\rightarrow$ & $1.224(1.024-1.463)$ & 0.026 \\
\hline & All-cause mortality or rehospitalisation & $\rightarrow-$ & $1.027(0.884-1.194)$ & 0.724 \\
\hline & CVD mortality & 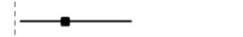 & $1.554(1.063-2.272)$ & 0.023 \\
\hline & Stroke & $\longrightarrow$ & $1.898(1.125-3.203)$ & 0.016 \\
\hline
\end{tabular}

B. Propensity Score Matched Cohort

\begin{tabular}{|c|c|c|c|c|}
\hline EF group & Outcomes & with $A F$ vs without $A F$ & HR $(95 \% \mathrm{CI})$ & $P$-value \\
\hline \multirow[t]{4}{*}{ Overall } & All-cause mortality & & $1.045(0.944-1.157)$ & 0.400 \\
\hline & All-cause mortality or rehospitalisation & & $0.991(0.912-1.076)$ & 0.829 \\
\hline & CVD mortality & $\rightarrow$ & $1.036(0.860-1.247)$ & 0.711 \\
\hline & Stroke & $\longrightarrow$ & $1.432(1.062-1.932)$ & 0.019 \\
\hline \multirow[t]{4}{*}{ HFrEF } & All-cause mortality & - & $1.017(0.886-1.167)$ & 0.810 \\
\hline & All-cause mortality or rehospitalisation & - & $0.971(0.871-1.083)$ & 0.188 \\
\hline & CVD mortality & -- & $0.914(0.716-1.166)$ & 0.470 \\
\hline & Stroke & $\longrightarrow$ & $1.557(1.032-2.348)$ & 0.035 \\
\hline \multirow[t]{4}{*}{ HFmrEF } & All-cause mortality & $\rightarrow$ & $1.083(0.809-1.451)$ & 0.590 \\
\hline & All-cause mortality or rehospitalisation & & $1.094(0.860-1.392)$ & 0.465 \\
\hline & CVD mortality & & $1.094(0.618-1.937)$ & 0.758 \\
\hline & Stroke & & $0.710(0.320-1.577)$ & 0.401 \\
\hline \multirow[t]{4}{*}{ HFpEF } & All-cause mortality & $\rightarrow-$ & $1.234(1.021-1.493)$ & 0.030 \\
\hline & All-cause mortality or rehospitalisation & $\rightarrow$ & $1.025(0.872-1.204)$ & 0.767 \\
\hline & CVD mortality & $\longrightarrow$ & $1.766(1.211-2.576)$ & 0.003 \\
\hline & Stroke & $\longrightarrow$ & $1.812(1.010-3.249)$ & 0.046 \\
\hline
\end{tabular}

Figure 4 Multivariable HR for adverse outcomes associated with $A F$, according to EF groups: $A$. unmatched cohort and B. propensity score matched cohort. AF, atrial fibrillation; CVD, cardiovascular disease; $E F$, ejection fraction; HFmrEF, heart failure with mid-range ejection fraction; HFpEF, heart failure with preserved ejection fraction; HFrEF, heart failure with reduced ejection fraction.

Table 3 Competing risk for CVD mortality and stroke according to AF in patients with HFrEF, HFmrEF and HFpEF

\begin{tabular}{|c|c|c|c|c|c|c|}
\hline & \multicolumn{2}{|l|}{ HFpEF } & \multicolumn{2}{|l|}{ HFmrEF } & \multicolumn{2}{|l|}{ HFrEF } \\
\hline & HR $(95 \%$ Cl) & $P$ value & HR $(95 \% \mathrm{Cl})$ & $P$ value & HR $(95 \% \mathrm{Cl})$ & $P$ value \\
\hline \multicolumn{7}{|l|}{ Stroke } \\
\hline Model 1 & $1.900(1.240$ to 2.910$)$ & 0.003 & $1.280(0.812$ to 2.020$)$ & 0.290 & 1.460 (1.040 to 2.050$)$ & 0.027 \\
\hline Model 2 & 1.766 (1.056 to 2.950$)$ & 0.030 & 1.120 (0.659 to 1.904$)$ & 0.680 & 1.544 (1.040 to 2.290$)$ & 0.031 \\
\hline \multicolumn{7}{|c|}{ CVD mortality } \\
\hline Model 1 & $1.210(0.892$ to 1.650$)$ & 0.220 & $0.956(0.658$ to 1.390$)$ & 0.810 & 0.882 (0.727 to 1.070$)$ & 0.200 \\
\hline Model 2 & 1.507 (1.005 to 2.260$)$ & 0.047 & 1.049 (0.634 to 1.736$)$ & 0.850 & 0.880 (0.685 to 1.130$)$ & 0.320 \\
\hline
\end{tabular}

Model 1: unadjusted.

Model 2: adjusted for sex, age, smoking status, alcohol consumption, medical history (New York Heart Association functional class, hypertension, diabetes mellitus, ischaemic heart disease, renal failure, cancer, prior stroke), heart rate, systolic blood pressure, diastolic blood pressure, body mass index, laboratory examination (white cell count, glycated haemoglobin, potassium, sodium, haemoglobin, blood urea nitrogen, creatinine, BNP or NT-proBNP), and medications before or at admission or at discharge (use of ACE inhibitor or angiotensin receptor blockers, beta-blockers, aldosterone antagonist, nitrate, diuretic, anticoagulant, antiplatelet and statin).

$\mathrm{AF}$, atrial fibrillation; BNP, brain natriuretic peptide; CVD, cardiovascular disease; HFmrEF, heart failure with mid-range ejection fraction; HFpEF, heart failure with preserved ejection fraction; HFrEF, heart failure with reduced ejection fraction; NT-proBNP, N-terminal prohormone of brain natriuretic peptide. 
types. Regarding the CV mortality, there was a significant interaction of diabetes in the HFpEF group, and patients without diabetes appeared to be more affected by AF. To the best of our knowledge, this study is the first to report the differential clinical impact of AF in East Asian patients with HFpEF, HFmrEF and HFrEF.

\section{$\mathrm{AF}$ and $\mathrm{HF}$ in Korea}

The prevalence of AF in patients with HF varies between different ethnic populations. ${ }^{18} 2425$ In this study of Korean patients with acute $\mathrm{HF}, 34.8 \%$ had $\mathrm{AF}$ at admission, which is similar to that reported in European patients with HF (30.3\%). ${ }^{18}$ The current study also demonstrated that the prevalence of $\mathrm{AF}$ increased with increasing $\mathrm{EF}$ and age, with patients in the HFpEF group aged 60-69 having the highest prevalence (49.5\%). This is in agreement with previous reports from other regions, which have shown the prevalence of AF in patients with HFrEF, HFmrEF and HFpEF to be $53 \%, 60 \%$ and $65 \%$, respectively, in the SwedeHF registry ${ }^{24} ; 27 \%, 29 \%$ and $39 \%$, respectively, in the European Society of Cardiology-Heart Failure (ESC-HF) longterm registry ${ }^{18}$; and $26.2 \%, 25.6 \%$ and $31.3 \%$ in the Canddesartan in Heart failure-Assessment of moRtality and Morbidity (CHARM) trial. ${ }^{25}$

$\mathrm{AF}$ and HF share common risk factors, the prevalence of which is increasing due to an ageing population and Westernisation of the lifestyle in Korea. Therefore, the prevalence of both $\mathrm{AF}$ and $\mathrm{HF}$ in Korea is expected to increase. ${ }^{6}$ Indeed, data have shown that the prevalence of AF increased from $0.36 \%$ in 2003 to $0.89 \%$ in $2013,{ }^{26}$ and that the prevalence of HF is expected to increase from $1.53 \%$ in 2013 to $3.35 \%$ by $2040 .{ }^{27}$ These results indicate that both $\mathrm{AF}$ and $\mathrm{HF}$ will represent a significant public health burden in the near future.

\section{Impact of AF on outcomes in HF}

AF has important clinical implications and can complicate HF by aggravating the condition and/or by increasing thromboembolic complications. HF is, therefore, a component of the $\mathrm{CHA}_{2} \mathrm{DS}_{2}$-VASc (congestive heart failure, hypertension, age $\geq 75$ years (double score), diabetes mellitus, previous stroke/ TIA (transient ischaemic attack) (double score), vascular disease, age 65-74 years, sex class (femalie)) score. ${ }^{28}$ The coexistence of $\mathrm{HF}$ and $\mathrm{AF}$ confers a substantially increased risk for CV morbidity and mortality. ${ }^{10}{ }^{29}$ Until now, the clinical implications of AF according to HF type have not been fully evaluated. The current study shows that AF had a differential effect on mortality depending on HF type, being associated with a $20 \%$ increased risk for all-cause and CV mortality only in patients with HFpEF and not in those with HFrEF and HFmrEF. The nationwide SwedeHF reported that AF was associated with similarly increased risks of death, HF hospitalisation, and stroke or TIA in all EF groups. ${ }^{24}$ By contrast, the ESC-HF long-term registry showed that AF was associated with poor outcomes in patients with HFpEF and HFmrEF, but not in those with HFrEF. ${ }^{18}$ The current study is more similar to that of the ESC-HF long-term registry and confirms a similar effect of $\mathrm{AF}$ on outcomes in an East Asian population for the first time. The reason for the increased mortality among patients with AF with HFpEF compared with other EF groups is not clear. Our speculation is that both AF and HFpEF share common risk factors, and although we performed propensity score matched analyses, unmeasured confounders or not-welldocumented risk factors may coexist and may confer excess mortality in patients with AF and HFpEF.
The most important complication of $\mathrm{AF}$ is stroke. As expected, AF was associated with a $54 \%$ and $94 \%$ increased risk for stroke in the HFrEF and HFpEF groups, respectively. One intriguing finding was the negative association between stroke risk and outcomes in the HFmrEF group, although the reason for this observation is not clear. Currently, $\mathrm{CHADS}_{2}$ (congestive heart failure, hypertension, age $\geq 75$ years, diabetes mellitus and previous stroke/TIA (double score)) and $\mathrm{CHA}_{2} \mathrm{DS}_{2}$-VASc scores do not differentiate between HFpEF and HFrEF. Because the impact of AF on stroke may be dependent on the HF type, further studies are required to determine whether the stroke risk scoring system should be revised to accommodate different weighting for each of the HF types.

This study has several limitations. First, because the KorAHF registry enrolled patients who were hospitalised for acute HF, our results may have overestimated the event rates for poor outcomes and may not be applicable to patients with chronic HF. Additional studies including patients with stable HF are required. Second, it is possible that there are additional potential confounders that were not accounted for in our study, although we adjusted for variables including medical history, signs and symptoms of HF, laboratory examinations, medications and device therapy.

Despite these limitations, this study had several strengths. First, to our knowledge, this study is the first to investigate the prognostic impact of AF in Korean patients with acute heart failure according to the three HF types. Second, the diagnosis of AF was determined according to an ECG interpreted by a cardiologist, indicating an accurate assessment of heart rhythm.

In conclusion, in East Asian patients with acute HF, AF was more common with increasing EF and was associated with increased mortality only in patients with HFpEF. AF was associated with an increased risk of stroke in both the HFrEF and HFpEF groups. These findings suggest the importance of AF management in patients with $\mathrm{HF}$ to minimise the risk of mortality and stroke.

Acknowledgements The Korean Acute Heart Failure (KorAHF) registry study was conducted in 10 tertiary medical centres: Seoul National University Hospital (Byung-Hee Oh), Seoul, Korea; Sungkyunkwan University College of Medicine (EunSeok Jeon), Seoul, Korea; University of Ulsan College of Medicine (Jae-Joong Kim), Seoul, Korea; Chungbuk National University College of Medicine (Myeong-Chan Cho), Cheongju, Korea; Kyungpook National University College of Medicine (Shung Chull Chae), Daegu, Korea; Catholic University of Korea (Sang Hong Baek), Seoul, Korea; Yonsei University College of Medicine (Seok-Min Kang), Seoul, Korea; Yonsei University Wonju College of Medicine (Byung-Su Yoo), Wonju, Korea; Seoul National University Bundang Hospital (Dong-Ju Choi), Seongnam, Korea; and Heart Research Center of Chonnam National University (Kye Hoon Kim), Gwangju, Korea.

Contributors MKS and JJP participated in the design of the study, performed the statistical analysis, interpreted the findings and drafted the manuscript. NKL contributed to the discussion. WHK and DJC participated in the design of the study, were involved in revising the manuscript for important intellectual content and provided final approval of the version to be published. All authors have read and approved the final manuscript.

Funding This work was supported by the Korea National Institute of Health intramural research grant 4800-4845-302 (2017-NI63001-00).

Competing interests None declared.

Patient consent for publication Not required.

Ethics approval The study protocol was approved by the ethics committee at each hospital, and the requirement for written informed consent was waived by the institutional review board.

Provenance and peer review Not commissioned; externally peer reviewed.

Data availability statement Data are available upon reasonable request. Legal restrictions prohibit us from making our data set publicly available. We were allowed to use the data from a South Korean government agency, Korea Centers for Disease 


\section{Key messages}

\section{What is already known on this subject?}

- The prognostic implications of atrial fibrillation (AF) in patients with heart failure (HF) remain controversial.

- The majority of current data suggest that $\mathrm{AF}$ is associated with increased mortality in patients with HF with preserved ejection fraction and $\mathrm{HF}$ with reduced ejection fraction.

- By contrast, the HF long-term registry of the European Society of Cardiology showed that AF was not associated with poor outcomes in patients with HF with reduced ejection fraction.

\section{What might this study add?}

- This study is the first to report the differential clinical impact of AF in East Asian patients with HF with preserved ejection fraction (HFpEF), $\mathrm{HF}$ with mid-range ejection fraction (HFmrEF) and HF with reduced ejection fraction (HFrEF).

- Data from the Korean Acute Heart Failure registry were evaluated, showing that the prevalence of AF increased with increasing ejection fraction in patients with acute heart failure.

- AF was seen to be associated with increased mortality in patients with HFpEF, but not in those with HFrEF and HFmrEF.

- AF was associated with an increased risk for stroke in patients with HFrEF and HFpEF.

\section{How might this impact on clinical practice?}

- The current study supports the prognostic value of evaluating AF status, when patients were stratified according to HF type, the novel aspect being the East Asian population.

- These findings suggest the importance of AF management in patients with HF to minimise the risk of mortality and stroke.

Control $(K C D C)$, on requested topics, and we are not allowed to open the data to the public yet. The KorAHF registry is strictly managed by KCDC, which provides the data to researchers at the contribution of each institution to the cohort enrolment. Those who are interested in related data may contact and request the data from KCDC in South Korea (email: jhkwh@nih.go.kr). Information from the KorAHF registry can be obtained from the following site: http://clinicaltrials.gov/ct2/show/NCT01389843.

Open access This is an open access article distributed in accordance with the Creative Commons Attribution Non Commercial (CC BY-NC 4.0) license, which permits others to distribute, remix, adapt, build upon this work non-commercially, and license their derivative works on different terms, provided the original work is properly cited, appropriate credit is given, any changes made indicated, and the use is non-commercial. See: http://creativecommons.org/licenses/by-nc/4.0/.

\section{ORCID iDs}

Won-Ho Kim http://orcid.org/0000-0002-4849-472X

Dong-Ju Choi http://orcid.org/0000-0003-0146-2189

\section{REFERENCES}

1 Kaarisalo MM, Immonen-Räihä P, Marttila RJ, et al. Atrial fibrillation and stroke. mortality and causes of death after the first acute ischemic stroke. Stroke 1997:28:311-5

2 Kannel WB, Wolf PA, Benjamin EJ, et al. Prevalence, incidence, prognosis, and predisposing conditions for atrial fibrillation: population-based estimates. Am J Cardiol 1998;82:2N-9.

3 Lip GYH, Rasmussen LH, Skjøth F, et al. Stroke and mortality in patients with incident heart failure: the diet, cancer and health (DCH) cohort study. BMJ Open 2012;2:e000975.
4 Alberts VP, Bos MJ, Koudstaal PJ, et al. Heart failure and the risk of stroke: the Rotterdam study. Eur J Epidemiol 2010;25:807-12.

5 Wolf PA, Abbott RD, Kannel WB. Atrial fibrillation as an independent risk factor for stroke: the Framingham study. Stroke 1991;22:983-8.

6 Batul SA, Gopinathannair R. Atrial fibrillation in heart failure: a therapeutic challenge of our times. Korean Circ J 2017:47:644-62.

7 Wang TJ, Larson MG, Levy D, et al. Temporal relations of atrial fibrillation and congestive heart failure and their joint influence on mortality: the Framingham heart study. Circulation 2003;107:2920-5.

8 Hu C-Y, Wang C-Y, Li J-Y, et al. Relationship between atrial fibrillation and heart failure. Eur Rev Med Pharmacol Sci 2016;20:4593-600.

9 Thihalolipavan S, Morin DP. Atrial fibrillation and heart failure: update 2015. Prog Cardiovasc Dis 2015;58:126-35.

10 Mamas MA, Caldwell JC, Chacko S, et al. A meta-analysis of the prognostic significance of atrial fibrillation in chronic heart failure. Eur J Heart Fail 2009;11:676-83.

11 Linssen GCM, Rienstra M, Jaarsma T, et al. Clinical and prognostic effects of atrial fibrillation in heart failure patients with reduced and preserved left ventricular ejection fraction. Eur J Heart Fail 2011:13:1111-20.

12 Banerjee A, Taillandier S, Olesen JB, et al. Ejection fraction and outcomes in patients with atrial fibrillation and heart failure: the Loire Valley atrial fibrillation project. Eur J Heart Fail 2012;14:295-301

13 McManus DD, Hsu G, Sung SH, et al. Atrial fibrillation and outcomes in heart failure with preserved versus reduced left ventricular ejection fraction. J Am Heart Assoc 2013:2:e005694.

14 Zakeri R, Chamberlain AM, Roger VL, et al. Temporal relationship and prognostic significance of atrial fibrillation in heart failure patients with preserved ejection fraction: a community-based study. Circulation 2013;128:1085-93.

15 Cheng M, Lu X, Huang J, et al. The prognostic significance of atrial fibrillation in heart failure with a preserved and reduced left ventricular function: insights from a metaanalysis. Eur J Heart Fail 2014;16:1317-22.

16 Lund LH, Donal E, Oger E, et al. Association between cardiovascular vs. noncardiovascular co-morbidities and outcomes in heart failure with preserved ejection fraction. Eur J Heart Fail 2014;16:992-1001.

17 Li S-J, Sartipy U, Lund LH, et al. Prognostic significance of resting heart rate and use of $\beta$-blockers in atrial fibrillation and sinus rhythm in patients with heart failure and reduced ejection fraction: findings from the Swedish heart failure registry. Circ Heart Fail 2015;8:871-9.

18 Zafrir B, Lund LH, Laroche C, et al. Prognostic implications of atrial fibrillation in heart failure with reduced, mid-range, and preserved ejection fraction: a report from 14964 patients in the European Society of Cardiology Heart Failure Long-Term Registry. Eur Heart J 2018;39:4277-84

19 Lee SE, Cho H-J, Lee H-Y, et al. A multicentre cohort study of acute heart failure syndromes in Korea: rationale, design, and interim observations of the Korean acute heart failure (KorAHF) registry. Eur J Heart Fail 2014;16:700-8.

20 Lee $\mathrm{SE}$, Lee $\mathrm{H}-\mathrm{Y}$, Cho H-J, et al. Clinical characteristics and outcome of acute heart failure in Korea: results from the Korean acute heart failure registry (KorAHF). Korean Circ J 2017:47:341-53.

21 Breslow N. Covariance analysis of censored survival data. Biometrics 1974;30:89-99.

22 S D. Partial residuals for the proportional hazards regression model. Biometrika 1982;69:239-41.

23 Fine JP, Gray RJ. A proportional hazards model for the Subdistribution of a competing risk. J Am Stat Assoc 1999;94:496-509.

24 Sartipy U, Dahlström U, Fu M, et al. Atrial fibrillation in heart failure with preserved, Mid-Range, and reduced ejection fraction. JACC Heart Fail 2017;5:565-74.

25 Lund LH, Claggett B, Liu J, et al. Heart failure with mid-range ejection fraction in CHARM: characteristics, outcomes and effect of candesartan across the entire ejection fraction spectrum. Eur J Heart Fail 2018;20:1230-9.

26 Son MK, Lim N-K, Park H-Y. Trend of prevalence of atrial fibrillation and use of oral anticoagulation therapy in patients with atrial fibrillation in South Korea (2002-2013) J Epidemiol 2018:28:81-7.

27 Lee JH, Lim N-K, Cho M-C, et al. Epidemiology of heart failure in Korea: present and future. Korean Circ J 2016:46:658-64

28 Camm AJ, Lip GYH, De Caterina R, et al. 2012 focused update of the ESC guidelines for the management of atrial fibrillation: an update of the 2010 ESC guidelines for the management of atrial fibrillation. developed with the special contribution of the European heart rhythm association. Eur Heart J 2012;33:2719-47.

29 Kotecha D, Chudasama R, Lane DA, et al. Atrial fibrillation and heart failure due to reduced versus preserved ejection fraction: a systematic review and meta-analysis of death and adverse outcomes. Int J Cardiol 2016:203:660-6. 